\title{
Development of the Vacuum Circuit Breaker with an Electromagnetic Actuator
}

\author{
Ayumu Morita Member (Hitachi Ltd., ayumu.morita.da@hitachi.com) \\ Masato Yabu Member (Hitachi Ltd., masato.yabu.jw@hitachi.com) \\ Satoru Kajiwara Member (Hitachi Ltd., satoru.kajiwara.fq@hitachi.com)
}

Keywords: vacuum circuit breaker, electromagnetic actuator, permanent magnet, solid lubricant, a minimum of maintenance

Recently, the development of vacuum circuit breakers (VCBs) using an electromagnetic actuator combined with a permanent magnet (PM) has attracted interest. This driving mechanism has the following advantages: (a) Simple structure due to significant reduction of the number of movable parts, (b) toughness and high reliability, (c) no complex mechanical latch needed because the closed position is held by the attractive force of the PM. Especially, simplification of the mechanism is expected to contribute to minimizing maintenance work.

This paper introduces our newly developed VCB with an electromagnetic actuator (Fig. 1). This VCB is characterized by the following: (a) a significantly simplified driving mechanism, (b) no need for grease, and (c) a new electromagnetic actuator called a hybridtype electromagnet. The driving mechanism consists of five units: an electromagnet (1), a trip spring (2), some auxiliary parts (3) (a mechanical auxiliary switch, a counter, and a position indicator), a control board (4), and a capacitor (5) to store the energy for opening and closing operations. The VCB is held in the closed position by attractive force of the PM without a coil current. The number of movable parts is decreased to $15 \%$ of that of our conventional product with a spring drive. Solid lubricant is used instead of grease for almost all of the linkages, which helps to eliminate periodical lubricating maintenance.

The hybrid-type electromagnet (Fig. 2) has an attractive force suitable for the spring force characteristics of the VCB through its combination of conventional plunger-type and plate-armature-type. Its core structure resembles that of the plunger-type near the open position, and becomes like that of the plate-armature-type when

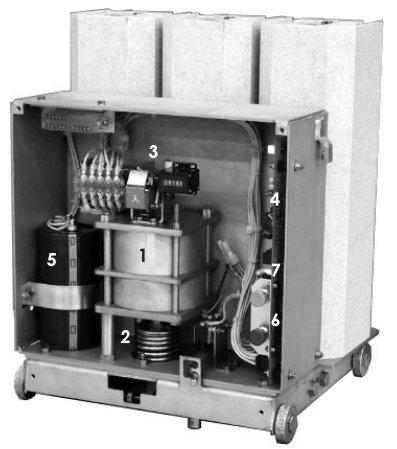

Fig. 1. Inside view of the mechanism housing (7.2 kV-600 A-12.5 kA VCB)

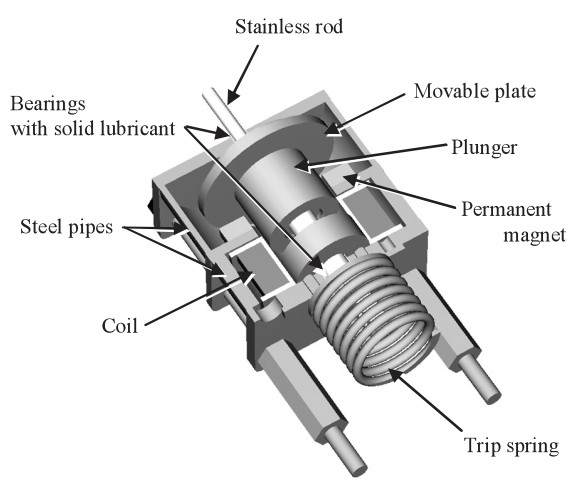

Fig. 2. Internal view of hybrid-type electromanget

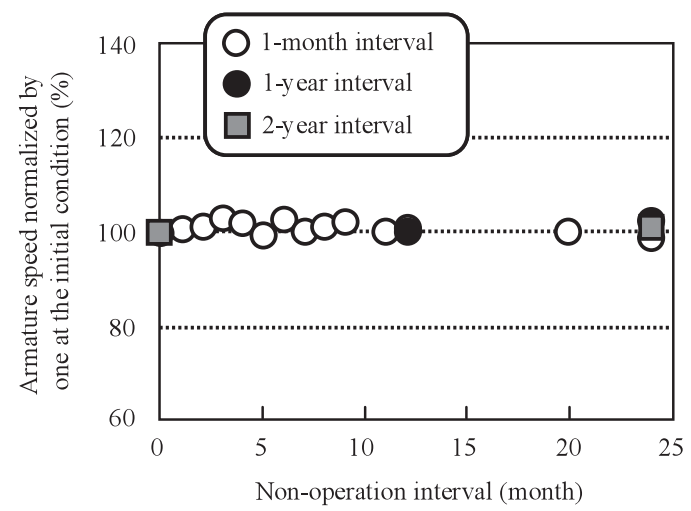

Fig. 3. Results of the rate operation test (Opening operation)

approaching the closed position. The PM is arranged outside the flux path of the magnet coil. It contributes to achieve long-term reliability of the PM because an inverse magnetic field working on the $\mathrm{PM}$ is very small when in the opening operation.

To assess the solid lubricant reliability, some special tests were performed in addition to the ordinary mechanical endurance test. We proposed a rare operation test to secure an important duty of circuit breakers to protect power circuits from a sudden fault. Dynamic characteristics were measured for three specimens at intervals of a month, a year, and two years, respectively. Experimental results are shown in Fig. 3. Variation of the armature speed is small and the influence of the non-operation interval is negligible. 


\title{
Development of the Vacuum Circuit Breaker with an Electromagnetic Actuator
}

\author{
Ayumu Morita* Member \\ Masato Yabu* Member \\ Satoru Kajiwara** ${ }^{* *}$ Member
}

A new type of vacuum circuit breaker (VCB) has been developed, which needs a minimum of maintenance. This VCB is characterized by the following: (a) a significantly simplified driving mechanism, (b) no need for grease, and (c) a new electromagnetic actuator called a hybrid-type electromagnet. The number of movable parts is decreased to $15 \%$ of that of our conventional product with a spring drive. Solid lubricant is adopted instead of grease for almost all of the linkages, which helps to eliminate periodical lubricating maintenance. The hybrid-type electromagnet has an attractive force suitable for the spring force characteristics of the VCB through its combination of conventional plunger-type and plate-armature-type electromagnet. The VCB is held in the closed position by the attractive force of the permanent magnet without using a coil current. Its core structure is optimized to prevent the permanent magnet from demagnetization and to secure long-term reliability. To assess the solid lubricant reliability, some special tests were performed such as a rare operation test. Dynamic characteristics were measured for three specimens at intervals of a month, a year, and two years, respectively. It was confirmed that variation of the armature speed is small and the influence of the non-operation interval is negligible.

Keywords: vacuum circuit breaker, electromagnetic actuator, permanent magnet, solid lubricant, a minimum of maintenance

\section{Introduction}

Vacuum circuit breakers (VCBs) dominate the medium voltage range from $1 \mathrm{kV}$ to $52 \mathrm{kV}$ for all power switching functions. In Japan particularly, VCBs occupy over $90 \%$ of the medium voltage market. VCBs have been developed in response to requirements for reductions in cost and size, environmental considerations, and improvements in safety and reliability. According to research of the Electric Technology Research Association, problems in the driving mechanism represent more than $50 \%$ of the VCB failure modes ${ }^{(1)}$. The major reason for mechanical trouble is hardening of the lubricating grease. The mechanism operates with linkages, levers, bearings, rollers, cams, springs and latches to close and open contacts. Although the best way to use VCBs long term is to perform complete cleaning and periodical lubrication, but this increases maintenance cost. In response to this, long life grease or solid lubricant such as $\mathrm{MoS}_{2}$ coating has been developed.

Recently, the development of VCBs using an electromagnetic actuator combined with a permanent magnet has attracted interest ${ }^{(2)-(5)}$. This driving mechanism has the following advantages:

- Simple structure due to significant reduction of the number of movable parts

- Toughness and high reliability

- No complex mechanical latch needed because the closed

\footnotetext{
* Power \& Industrial Systems R\&D Lab, Power Systems, Hitachi Ltd.

1-1-1, Kokubucho, Hitachi 316-8501

** Hitachi Works, Power Systems, Hitachi Ltd.

1-1-1, Kokubucho, Hitachi 316-8501
}

position is held by the attractive force of the permanent magnet.

The permanent magnet and the aluminum electrolysis capacitor are key technologies of the new driving mechanism. A neodymium iron boron magnet $(\mathrm{NdFeB})$ has dramatically increased magnetic energy compared with conventional magnets and its price is anticipated to decrease with increasing demand and production amount. The reliability of the electrolysis capacitor has been improved recently as its market has been expanded to include utilization in the inverter circuit of hybrid cars. In the magnetic actuator, the capacitor helps to store closing and opening operation energy. In this usage a ripple current causing internal heating is negligible. A lifetime of the capacitor is expected over 20 years according to the Arrhenius's equation. The capacitor power supply solves the following technical problems in the conventional DC directly applied magnetic drive that 1) a large power supply or battery is necessary to produce a high ampere-turn and 2) dynamics of the contact in the closing and opening operations is influenced by fluctuation of the control voltage.

This paper presents our newly developed VCB with an electromagnetic actuator, called a hybrid-type electromagnet, which is suitable for the spring force characteristics of the VCB. In addition, using a solid lubricant technique, we achieved a minimum of maintenance. To assess the solid lubricant reliability, we performed some special tests such as a rare operation test in addition to the ordinary mechanical endurance test.

\section{Structure of the New VCB}

Figure 1 shows our new VCB with a rating of 7.2 
$\mathrm{kV}-600 \mathrm{~A}-12.5 \mathrm{kA}$. The driving mechanism of the VCB consists of five units: an electromagnet (1), a trip spring (2), some auxiliary parts (3) (a mechanical auxiliary switch, a counter, and a position indicator), a control board (4), and a capacitor (5) to store the energy for opening and closing operations. The VCB is held in the closed position by attractive force of the permanent magnet without a coil current. The number of movable parts is significantly decreased to $15 \%$ of that of our conventional product with a spring drive. The dimensions of the VCB are set equal to that of our conventional product to secure interchangeability.

When the capacitor is sufficiently charged for operation with a small charge power of less than DC100 V/1 A, an LED (7) lights up as a sign of "ready-to-close." The VCB can be operated by a remote signal or pressing a push-button (6) locally. When the external power supply is shut down, only the opening operation can be performed by the push-button within the first 10 minutes using stored energy in the capacitor. After 10 minutes, an emergency manual lever must be used. For the magnetic drive, it is one of the weak points not to be able to do a closing operation manually. It is also inconvenient during check and maintenance work. A handy power unit using dry-cell batteries is prepared for maintenance work as shown in Fig. 2.

Solid lubricant is used instead of grease for almost all linkages, which helps to eliminate periodical lubricating maintenance. A stainless steel rod is used as the axis of the linkage. The bearing consists of a lubricating layer and bronze

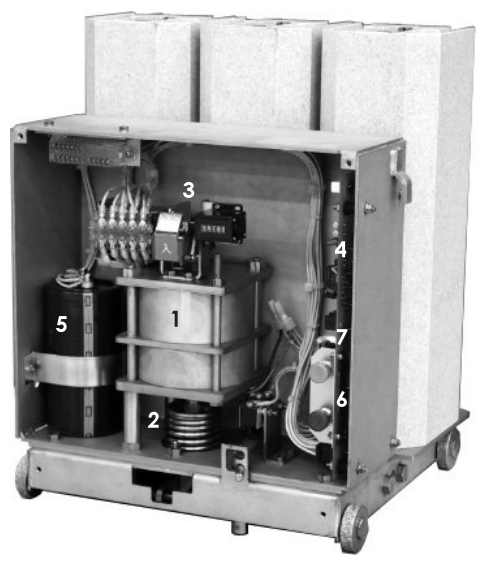

Fig. 1. Inside view of the mechanism housing (7.2 kV-600A-12.5 kA VCB)

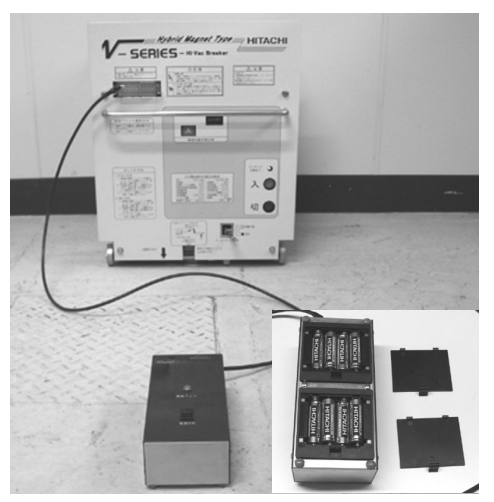

Fig. 2. Handy power unit for maintenance work sintered on the surface of a steel base. The lubricating layer is a compound material of solid lubricating powder and polytetrafluoroethylene (PTFE). PTFE has a low friction coefficient of $\mu=0.03-0.2$ and is stable chemically. We confirmed that over 100,000 closing and opening operations could be made in the mechanical endurance test.

\section{Hybrid-type Electromagnet}

3.1 Concept As is well known, electromagnets can be divided roughly into two types, a plunger-type (Fig. 3(a)) and a plate-armature-type (Fig. 3(b)). The plunger-type electromagnet is suitable for long-stroke driving due to its moderately small magnetic resistance. On the other hand, in the plate-armature-type electromagnet, the attractive force works at both the center and side cores, so it is stronger than that of the plunger-type. Our proposed electromagnet, a hybrid-type in Fig. 3(c), is based on a combined concept of the plungertype and the plate-armature-type. The armature of the hybridtype electromagnet consists of a plunger and a movable plate, each connected like the letter T. Figure 4 illustrates comparison of the attractive force among these electromagnets, which is calculated numerically by the finite element analysis program described in Section 3.3. The following calculation conditions are set equal for each electromagnet: the sectional area of the core, the capacitance and charging voltage of the capacitor power supply, and the turn number and resistance of the coil windings. Although the plate-armature-type electromagnet cannot be applied to the long-stroke driving, a higher attractive force is achieved in case of the short-stroke one. The attractive force feature of the hybrid-type electromagnet resembles that of the plunger-type near the open position, and becomes like that of the plate-armature-type when approaching the closed position. It is fit for the spring force characteristics of the VCB, which steeply increase at the contact touch position due to the contact pressure springs.

3.2 Structure and Operation Internal structure of

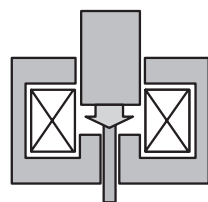

(a) Plunger-type

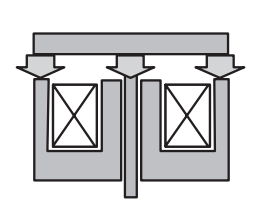

(b) Plate-armature-type

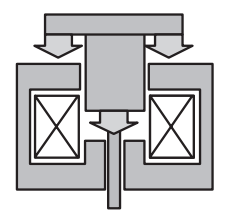

(c) Hybrid-type
Fig. 3. Types of the electromagnets

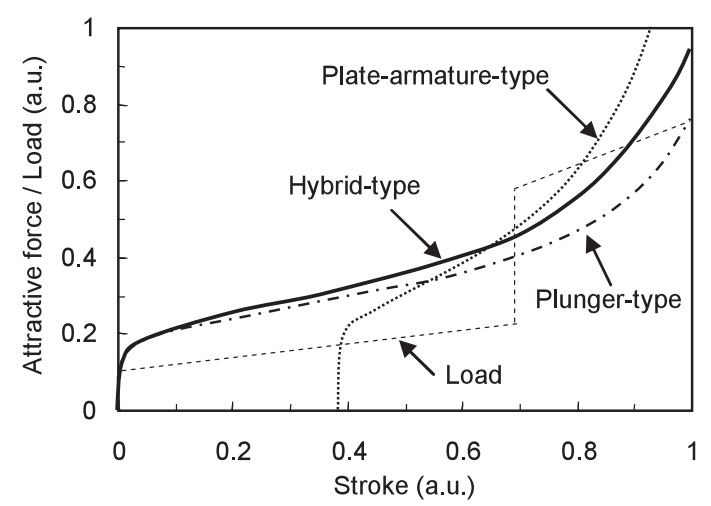

Fig. 4. Comparison of the attractive force with the VCB spring force characteristics 
the new actuator is illustrated in Fig. 5. The stationary core consists of steel plates and standard sized steel pipes. The armature is composed of a stainless steel rod, a plunger and a movable plate facing a permanent magnet. Bearings with solid lubricant are used to guide the armature movement. External magnetic flux leaked from the actuator is very small with a magnetic flux density of less than $20 \mathrm{mT}$, because the electromagnet is entirely covered with the magnetic material.

In the following, the operation sequence of the actuator is explained using Fig. 6. Figure 6(a) illustrates the closing operation. The vacuum interrupters are kept in the open position by the trip spring force. When a closing signal is given to the control board, the energy stored in the capacitor is transferred to the electromagnet. The attractive force works on the plunger, and the armature starts to move downwards. When the armature is approaching the closed position, the magnetic flux of the permanent magnet is superimposed on that of the electromagnet. As mentioned above, the driving force of the actuator is suitable for the VCB spring force, because the attractive force is steeply increased near the contact touch position.

As shown in Fig. 6(b), the vacuum interrupters are kept in the closed position by only the attractive force of the permanent magnet. The holding force is set to be higher than that

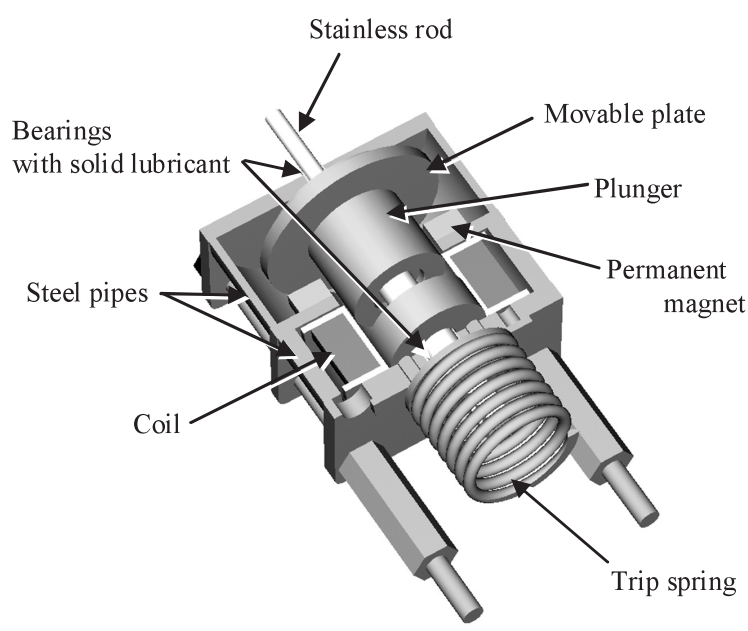

Fig. 5. Internal structure of the hybrid-type electromanget of the contact pressure springs and the trip spring, considering the effect of an external shock/vibration such as from an earthquake.

In the opening operation, the electromagnet is inversely magnetized as shown in Fig. 6(c). An inverse magnetic flux is imposed on that generated by the permanent magnet which weakens its holding force. The armature starts to move upwards. The opening velocity of the armature is determined mainly by the energy of the contact pressure springs and the trip spring charged in the closing operation.

The permanent magnet is arranged outside the flux path of the magnet coil. It contributes to minimize an ampere-turn in the closing operation because the permeability of the permanent magnet is roughly equal to that of the air gap. In addition, in the opening operation, a very small inverse magnetic field works on the permanent magnet as shown in Fig. 6(c). It is important to achieve long-term reliability of the permanent magnet.

3.3 Numerical Analysis To design the actuator, we utilized a finite element analysis program. This program can perform nonlinear transient electromagnetic analysis coupled with a dynamic solution. It can also deal with a capacitor power supply. Figures 7(a), (b) and (c) give the results of the magnetic field analysis in the closing operation, holding the closed position and the opening operation, respectively. The comparison with analytical and experimental results is shown in Fig. 8. In both cases of closing and opening operations, the analytical results are in relatively good agreement with the experimental ones. The experimental stroke curve in the opening operation has some inflection points, which are caused by the moving mass non-continuity at the contact touch position, the bending of the base and/or case in holding the closed condition, and so on. However, we don't think the precision of the contact movement so important. In designing the driving mechanism of the $7.2 \mathrm{kV}$ class VCB, we focus on not the contact speed but the contact welding when in closing and carrying a short-circuit current. In the opening operation, our driving mechanism is actuated mainly by the contact pressure and trip springs. To achieve the short-current closing and carrying ability, we set the following parameters to the same as those of the conventional models: (i) the force

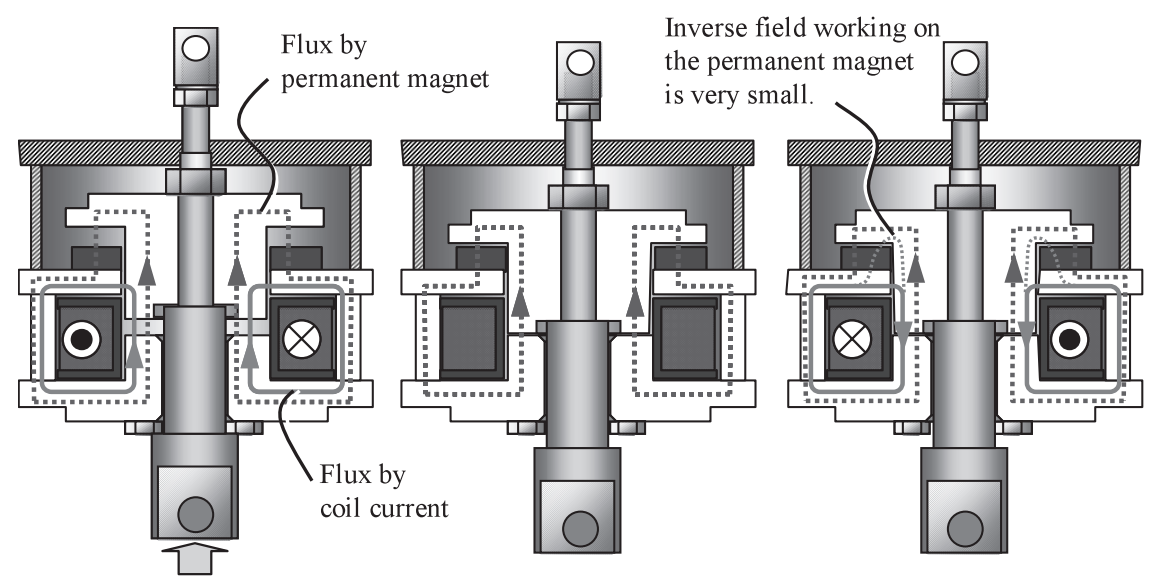

Trip spring force

(a) Closing operation (b) Holding the closed position

Fig. 6. Principle of the operations (c) Opening operation 


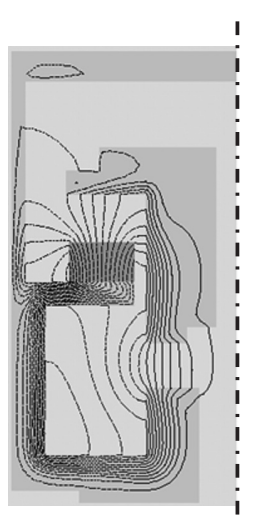

(a) Closing operation

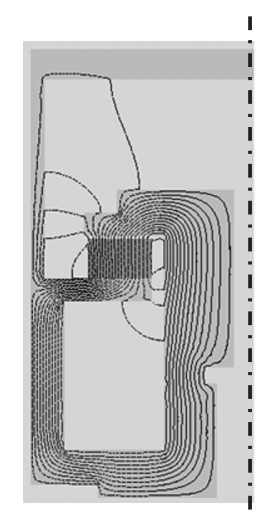

(b) Holding the closed position

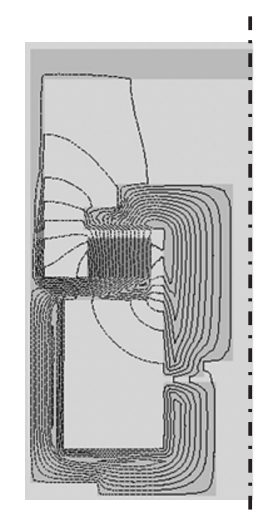

(c) Opening operation
Fig. 7. Results of the numerical analysis
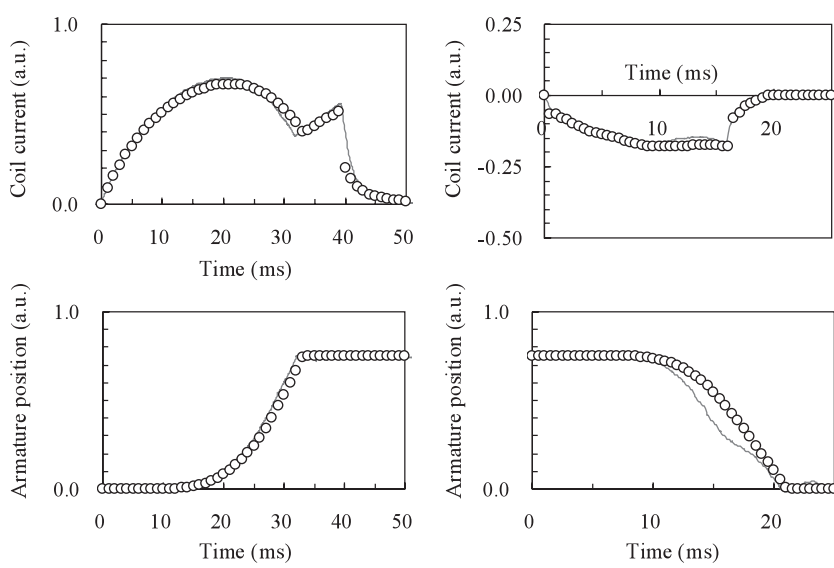

(a) Closing operation

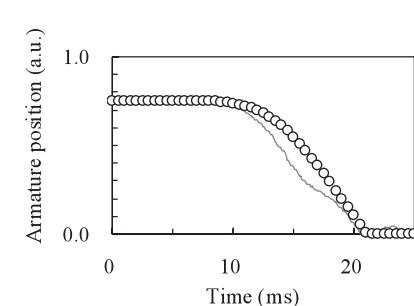

(b) Opening operation

Fig. 8. Comparison with analytical (circular symbols) and experimental results (solid lines) for closing and opening operations

of the contact pressure springs to decrease the contact welding force and (ii) the stored energy of the contact pressure and trip springs to break the weld in the opening operation.

3.4 Non-demagnetized Structure of the Permanent Magnet One of the most remarkable characteristics of the hybrid-type electromagnet is to prevent the permanent magnet from demagnetization. Factors affecting the permanent magnet stability include time, reluctance changes, external adverse field, mechanical shock/stress, and so on. Time degradation of the permanent magnet is known to be minimal with a flux loss per 30 years of less than a few percent. Also we have confirmed that the effect of mechanical shock and stress is negligible based on the VCB endurance test with over 100,000 closing and opening operations. The effect of the reluctance changes and the external adverse field can be evaluated based on the operating point behavior in the second quadrant of the $B-H$ curve (demagnetization curve). A permanent magnet has a static operating point if air-gap dimensions are fixed. This operating point moves along the demagnetization curve when the external magnetic field is applied. Figure 9 shows the operating point behavior in closing and opening operations from the numerical analysis. In the hybrid-type electromagnet, the adverse magnetic field strength applied in the operation is suppressed much smaller than the coercive force of the permanent magnet, $H_{c}$.

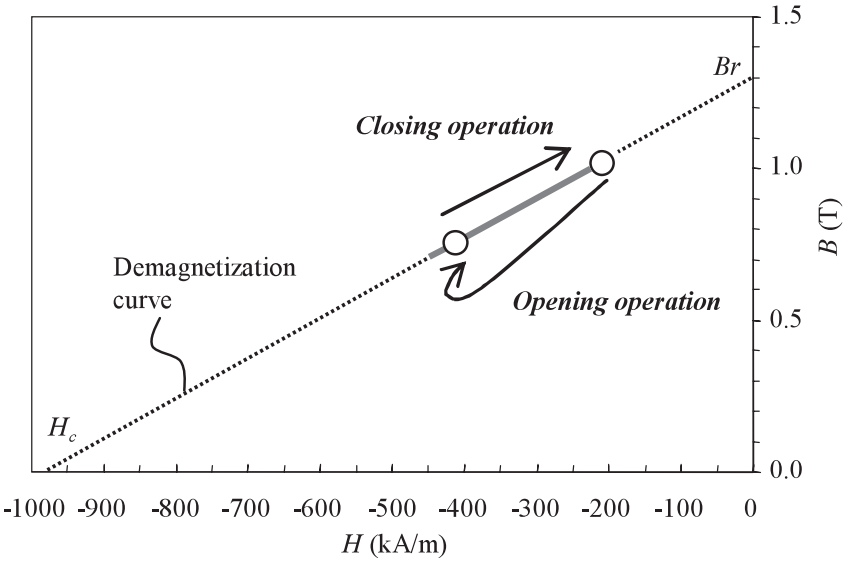

Fig. 9. Operating point behavior of the permanent magnet in the closing and opening operations

\section{Reliability Assessments of the New Mecha- nism}

4.1 Conventional Endurance Test To assess reliability of the newly developed mechanism, we performed several kinds of tests. First we did a conventional endurance test. The test consisted of more than 10,000 operating sequences without any maintenance. Using an automatic testing apparatus (Fig. 10), we could check the mechanical travel characteristics every operation. Measured parameters were armature travel curve, coil current, operating time and speed, contact chattering time of the main and auxiliary switches, and so on. The variation between the values of each parameter during the test is within our tolerance of $5 \%$. We extended the test up to 100,000 operations with some maintenance only to the electric parts such as the auxiliary switch, and we confirmed that the mechanical parts including solid lubricant linkages have enough durability.

In addition, we performed the following special examinations to consider various circumstances and operating conditions.

4.2 Low and High Temperature Tests JEC standard, JEC-2300-1998, defines the ambient air temperature at normal service conditions as -20 to $+40^{\circ} \mathrm{C}$. The maximum temperature was set to $+60^{\circ} \mathrm{C}$ in this test, because we considered a temperature rise in the cubicle or switchgear of $15 \mathrm{~K}$ and a tolerance of $5 \mathrm{~K}$. As shown in Fig. 11, the influence of the ambient temperature is small and the variation of the armature speed is within 5\%. The armature speed in the opening operation slightly increases in proportional to the temperature, because the temperature rise weakens the attractive force of the permanent magnet.

4.3 Considerations of Circumstances A salt spray test was made to evaluate the corrosion resistance to salt mist of the solid lubricant linkages. The entire mechanism was sprayed with a salt mist (density of 5\%) once and kept for one month (Fig. 12(a)). This density is equivalent to that in a heavy pollution condition in accordance with the JIS standard. Although the steel base of the bearings was rusted and corroded, the solid lubricant layer was stable. The variation between mechanical travel characteristics measured before and after the test is within our tolerance of $5 \%$. 


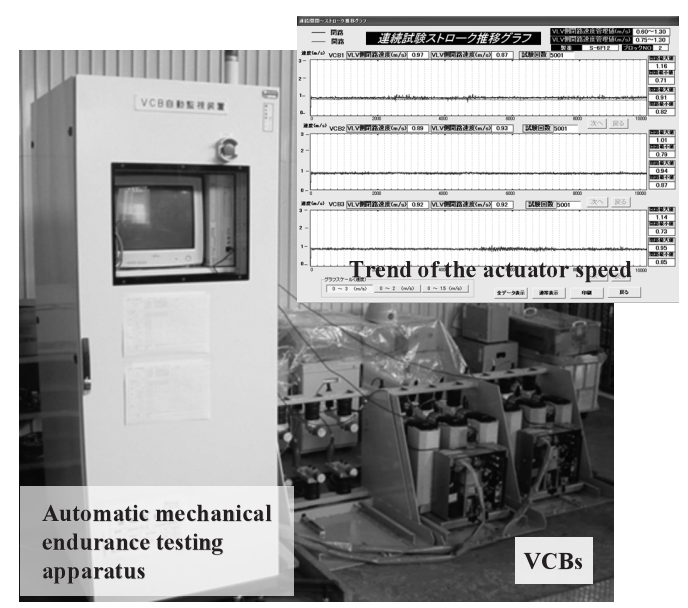

Fig. 10. Automatic mechanical endurance testing apparatus

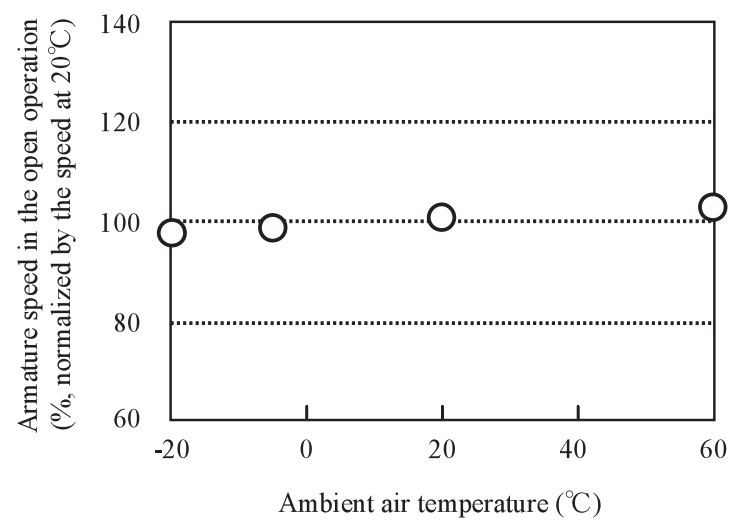

Fig. 11. Influence of the ambient air temperature on the armature speed in the opening operation

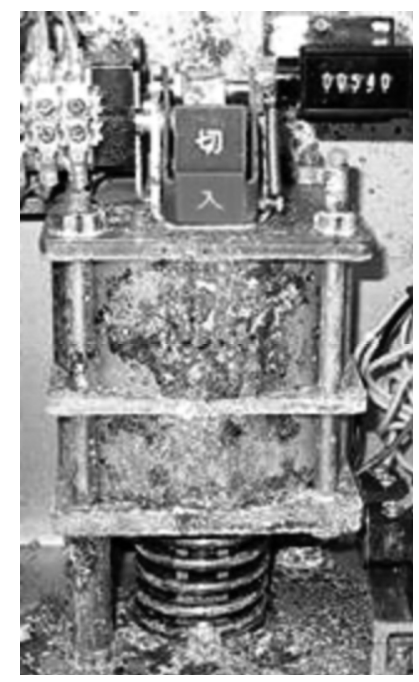

(a) Salt spray test

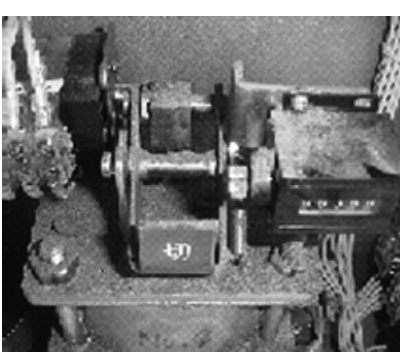

(b) Steel dust test
Fig. 12. Photographs of the magnetic actuator after circumstance consideration tests

The new actuator has a permanent magnet internally. Although magnetic flux density leaked from the actuator is small, it can attract magnetic dust. As shown in Fig. 12(b), we sprinkled a large amount of steel dust on the actuator and checked the mechanical travel characteristics. We found that the variation of the characteristics is negligible during $50 \mathrm{op}-$ erations and the dust did not intrude into the actuator.
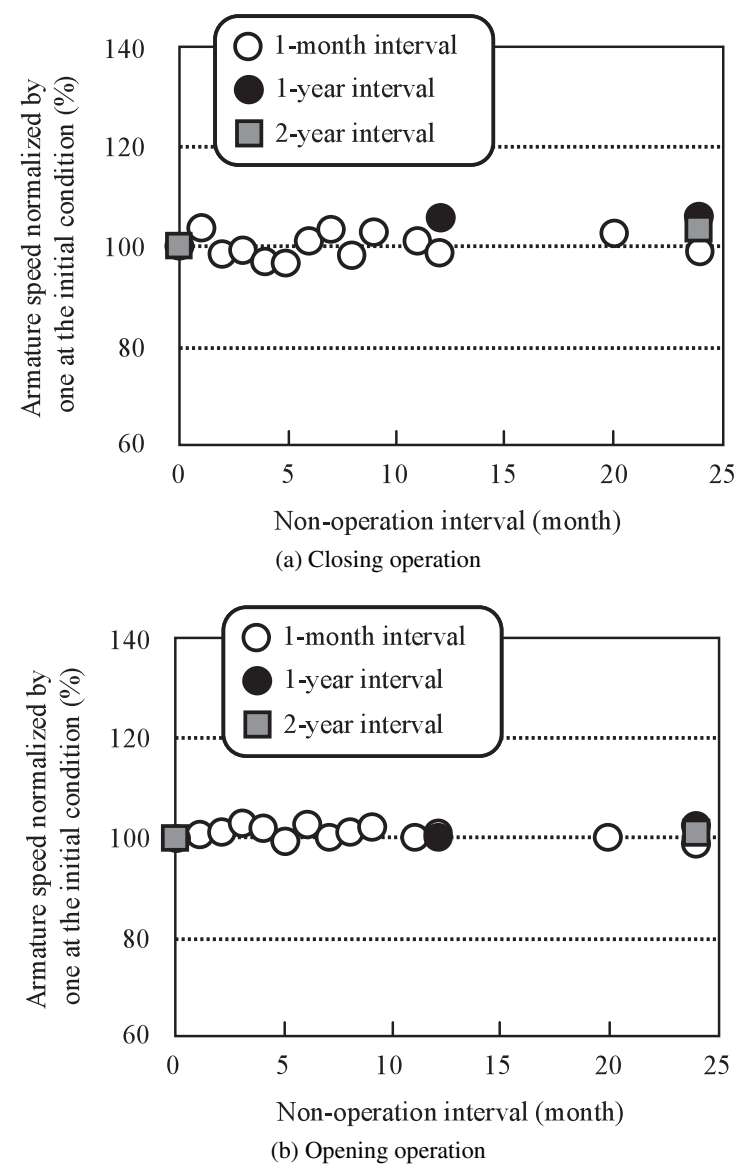

Fig. 13. Results of the rare operation test

4.4 Rare Operation Test Circuit breakers have an important duty to protect power circuits from a sudden fault. For the mechanism, experiencing a sudden operation is much severer than experiencing a continuous one. The major reason for mechanical failure, hardening of the grease, is due to the rare operation duty. Influence of the non-operation interval on the mechanical travel characteristics was investigated. For three VCBs, non-operation intervals were set to be a month, a year and two years, respectively. These specimens were kept in the machining factory, in which a large amount of metal dust is present in the atmosphere. Experimental results are shown in Fig. 13. Variation of the armature speed is small and the influence of the non-operation interval is negligible.

\section{Lineup Developments}

We have been developing lineups of the new VCBs as shown in Table 1. Three sizes of actuators are being fabricated according to the rated short-circuit breaking current. We note that the outer dimensions of the class II actuator are almost equal to those of the class I actuator to achieve interchangeability with our conventional spring drive product. Because the class II actuator requires a holding force and a driving force 1.5 times those of the class I actuator, the plunger and the permanent magnet are scaled up proportionally.

For the class III actuator, shortening the closing and opening times is the most important technical theme. As the actuator is scaled up, the coil inductance and the required 
Table 1. VCB lineups using a electromagnetic actuator

\begin{tabular}{|c|c|c|c|c|c|c|c|c|}
\hline & \multicolumn{7}{|c|}{ Rated short circuit current $(\mathrm{kA})$} \\
\hline & & 8 & 12.5 & 16 & 20 & 25 & 31.5 & 40 \\
\hline \multirow{2}{*}{ 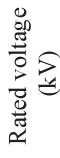 } & 3.6 & 0 & 0 & 0 & 0 & 0 & 0 & 0 \\
\hline & 7.2 & 0 & 0 & & 0 & & 0 & 0 \\
\hline \multirow{5}{*}{ 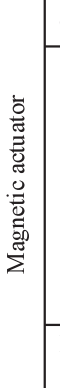 } & Class & \multicolumn{3}{|c|}{ I } & \multicolumn{2}{|c|}{ II } & \multicolumn{2}{|c|}{ III } \\
\hline & \multirow{3}{*}{ 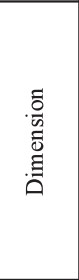 } & \multicolumn{3}{|c|}{ 㱏 } & \multicolumn{2}{|c|}{ 量 } & \multicolumn{2}{|c|}{ 요 } \\
\hline & & \multicolumn{3}{|c|}{ 直 } & \multicolumn{2}{|c|}{ 首 } & \multirow{2}{*}{\multicolumn{2}{|c|}{$\begin{array}{c}\text { Dase area } \\
3 S \\
3 S\end{array}$}} \\
\hline & & \multicolumn{3}{|c|}{$\begin{array}{l}\text { Base area } \\
\quad S\end{array}$} & \multicolumn{2}{|c|}{$\begin{array}{c}\text { Base area } \\
S\end{array}$} & & \\
\hline & $\begin{array}{l}\text { Force } \\
\text { ratio }\end{array}$ & \multicolumn{3}{|c|}{2} & \multicolumn{2}{|c|}{3} & \multicolumn{2}{|c|}{10} \\
\hline
\end{tabular}

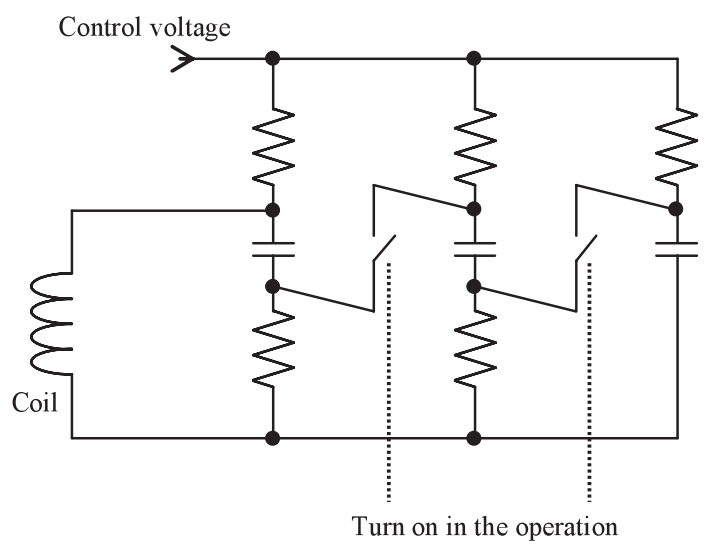

Fig. 14. Parallel-charge and series-discharge technique

input power increase. Response time is proportional to $\sqrt{L C}$, where $L$ and $C$ are the coil inductance and the capacitance of the power supply, respectively. We have proposed the "parallel-charge and series-discharge" of the capacitors as illustrated in Fig. 14. For closing and opening operations, the connection of some capacitors is converted from parallel to series. As shown in Table 1, the outer dimensions of the class III actuator are scaled up roughly three times those of the class II actuator. In addition, the class III actuator requires a stored energy three times as high as that in the class II actuator. By applying this technique, the response time does not change in principle, based on the equation, $\sqrt{3 L \times C / 3}=\sqrt{L C}$. This method makes it possible to shorten the breaking time of the class III VCB to within $50 \mathrm{~ms}$ (three cycles at $60 \mathrm{~Hz}$ ).

\section{Conclusions}

In this paper, we introduced our newly developed VCB with a magnetic drive. The most remarkable feature of our development is use of a solid lubricant to eliminate the need for periodical lubricating maintenance. To assess the reliability of the new lubricant technique, we performed some special tests in addition to the ordinary mechanical endurance test. A rare operation test was proposed to secure protection of power circuits from a sudden fault. We have confirmed the high reliability of our VCB from the test results. The magnetic drive also has a new actuator called a hybrid-type electromagnet, which has a good attractive force suited to the spring force characteristics of the VCB. This actuator secures long-term reliability by optimizing its core structure to prevent the permanent magnet from demagnetization. Advantages of the magnetic drive include a simpler structure, better toughness, higher reliability, and a minimum of maintenance in comparison with the conventional spring drive. We anticipate that the magnetic driving mechanism should become the mainstream mechanism for VCBs.

(Manuscript received March 14, 2006, revised July 27, 2006)

\section{References}

(1) Electric Research Technology (Denki-Kyoudo-Kenkyuu), Vol.56, No.2 (2000)

( 2 ) E. Dulluni: "A vacuum circuit-breaker with permanent magnetic actuator for frequent operations", Proc. ISDEIV. XVIIIth International Symposium, Vol.2, pp.688-691 (1998)

( 3 ) S.A. Ruhland: "Vacuum circuit breaker with asymmetrical actuator", Transmission and Distribution Conference and Exhibition 2002: Asia Pacific. IEEE/PES, Vol.2, pp.909-913 (2002)

( 4 ) J. Sato, O. Sakaguchi, N. Kubota, S. Makishima, S. Kinoshita, T. Shioiri, T. Yoshida, M. Miyagawa, M. Homma, and E. Kaneko: "New technology for medium voltage solid insulated switchgear", Transmission and Distribution Conference and Exhibition 2002: Asia Pacific. IEEE/PES, Vol.3, pp.17911796 (2002)

( 5 ) T. Takeuchi, T. Nakagawa, M. Tsukima, K. Koyama, N. Tohya, and T. Yano: "An Electromagnetically Actuated Vacuum Circuit Breaker Developed by Electromagnetic Analysis Coupled with Motion", IEEJ Trans. PE, Vol.124, No.2, pp.321-326 (2004)
Ayumu Morita

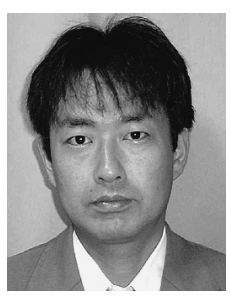

Masato Yabu (Member) He was born in Yokohama, Japan, on June

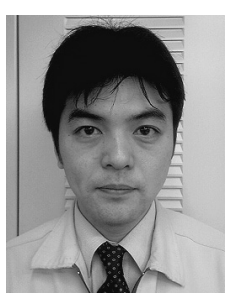

Satoru Kajiwara

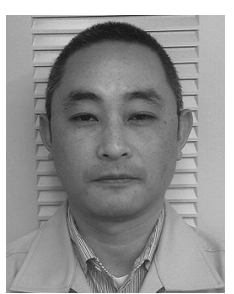
30, 1974. He received his bachelors degree in Physics
in 1998 from Sophia University, Japan. He joined Kokubu Works, Hitachi Ltd in 1998, where he has been engaged mainly in the development of vacuum circuit breakers. He is a member of IEEJ.
(Member) He was born in Ibaraki, Japan, on August 21, 1967. He received his bachelors degree in 1990 and his doctorate in electrical engineering in 1995 from the University of Tokyo, Japan. He joined Power \& Industrial Systems R \& D Laboratory, Hitachi Ltd in 1995, where he has been engaged in the research and development of mediumvoltage switchgear and vacuum circuit breakers. He is a member of IEEJ, the American Physical Society, and the Vacuum Society in Japan. 